\title{
Lotta alla plastica ed ecosistemi marini. II quadro giuridico all'indomani della direttiva UE/2019/904
}

\section{Premessa}

Una delle più grandi sfide ambientali della nostra epoca è senz'altro rappresentata dalla "lotta alla plastica".

Tale materiale ha, infatti, nella nostra realtà, un enorme peso: sociale, in quanto aiuta la società ad affrontare le sfide contemporanee ${ }^{1}$, ed economico, dato che genera un'industria particolarmente rilevante nell'economia europea ${ }^{2}$.

È addirittura terzo per diffusione sulla terra, dopo cemento e acciaio, rinvenendosi pressoché ovunque, dalle più estreme profondità oceaniche fino all'aria che respiriamo.

Viene infatti prodotto - e ciò già a partire dagli anni Cinquanta del secolo scorso - in maniera indiscriminata e non ecosostenibile; viene consumato, di

${ }^{1}$ La multifunzionalità, infatti, rende tale materiale particolarmente utile al fine di affrontare diverse sfide della nostra società: si pensi all'utilizzo di materiali plastici leggeri e innovativi nelle automobili o negli aerei, che consente di risparmiare carburante e ridurre le emissioni di $\mathrm{CO}_{2}$; si pensi, ancora, agli imballaggi di plastica, che garantiscono la sicurezza degli alimenti e riducono i rifiuti alimentari; si pensi, di nuovo, che, in combinazione con la stampa $3 \mathrm{D}$, le materie plastiche biocompatibili possono salvare vite umane, rappresentando un esempio di innovazione in campo medico. In tal senso, cfr. COM (2018) 28 final.

${ }^{2}$ Cfr. COM (2018) 28 final, ove si rileva che "nel corso degli ultimi 50 anni il ruolo e l'importanza della plastica nella nostra economia sono cresciuti in modo costante. La produzione mondiale di plastica è aumentata di venti volte rispetto agli anni ' 60 del secolo scorso, raggiungendo 322 milioni di tonnellate nel 2015, e nei prossimi 20 anni dovrebbe raddoppiare. Nell'UE il settore della plastica dà lavoro a 1,5 milioni di persone e nel 2015 ha generato un giro d'affari di 340 miliardi di euro". 
frequente, in prodotti mono-uso, "usa e getta", senza che vi sia riutilizzazione o riciclo; infine, viene smaltito e disperso nell'ambiente, compreso quello marino, minacciando l'intero ecosistema e la sopravvivenza di numerosi animali acquatici e minando - con l'ingresso nella catena alimentare - anche la salute di chi, come l'uomo e i volatili, si nutre di quelle specie.

Il presente lavoro intende, nei paragrafi che seguono, illustrare il terribile impatto ambientale connesso al consumo lineare della plastica monouso, così da poter descrivere, nel proseguo, gli obiettivi enunciati e le soluzioni giuridiche offerte dall'Unione europea.

\section{Le origini del problema: gli effetti della produzione indiscriminata e del consumo lineare della plastica sull'ecosistema marino e sulla salute collettiva}

Nell'attuale contesto storico, la plastica - e in particolare la sua produzione, il suo consumo e il suo smaltimento - ha un considerevole impatto negativo sull'ambiente e sulla salute umana e animale ${ }^{3}$.

La causa principe di tale impatto negativo risiede nei caratteri di tale materiale, che, da una parte, è leggero, multifunzionale e poco costoso -caratteri che rendono elevati la sua produzione ${ }^{4}$ e il suo utilizzo - dall'altra, è riconducibile ad un modello economico lineare, essendo assai diffuso l'impiego, specie nei prodotti di rapido consumo, della plastica monouso, assai raramente riutilizzabile e riciclabile, e invece da smaltire, con dispersione nell'ambiente, al termine del suo ciclo di vita ${ }^{5}$ (si pensi - per fare qualche esempio - ai contenitori in plastica, alle borse, alle tazze, ai coperchi, alle cannucce, alle posate, ai piatti, e via dicendo).

${ }^{3}$ Per alcune letture relative agli effetti del consumo lineare della plastica nei mari, cfr. E. Nicoli, C. Spadaro, P. Antonelli, Plastica addio. Fare a meno della plastica: istruzioni per un mondo e una vita "zero waste", Milano 2019; W. McCallum, Vivere senza plastica, Milano 2019. Cfr. altresì, i due report pubblicati dal WWF, rispettivamente nel 2018 e 2019, dal titolo "Mediterraneo in trappola. Come salvare il mediterraneo dalla plastica" e "Fermiamo l'inquinamento da plastica. Italia: una guida pratica per uscire dalla crisi della plastica".

${ }^{4}$ Cfr. COM (2018) 28 final, che spiega come tale produzione mondiale sia sempre in crescita, sia aumentata di ben 20 volte negli ultimi cinquant'anni e sia stimata in 322 tonnellate annuali (e di queste, 25,8 milioni di tonnellate vengono prodotte in Europa). Di tale numero, poi, il $39,9 \%$ è prodotto per gli imballaggi, il $19,7 \%$ nell'edilizia, $1{ }^{\prime} 8,9 \%$ nel settore automobilistico e il $5,8 \%$ nell'elettronica.

${ }^{5}$ Cfr. COM (2018) 28 final, che evidenzia come il riutilizzo e il riciclaggio della plastica a fine vita siano oggi molto bassi: dei 25,8 milioni di tonnellate di rifiuti di plastica generati ogni anno in Europa, solo il $30 \%$ viene raccolto per essere riciclato.

Tale comunicazione mette altresì in luce che "la domanda di plastica riciclata oggi rappresenta solo il $6 \%$ circa della domanda di plastica in Europa [...]. Gli investimenti in nuova capacità di riciclaggio della plastica sono stati frenati dalle prospettive di bassa redditività del settore". Tuttavia, 
Parte di tale dispersione colpisce anche l'ambiente marino.

A livello mondiale, ogni anno, infatti - per fornire qualche dato - finiscono negli oceani da 5 a 13 milioni di tonnellate di plastica; e, di tale quantità, in Europa, ne approdano in mare da 150 a 500 mila tonnellate. Tale ingente quantità di plastica rappresenta addirittura più del $80 \%$ dei rifiuti marini complessivamente rinvenuti sulle spiagge, di cui, poi, il $50 \%$ è costituito da oggetti di plastica "usa e getta" e il $27 \%$ da attrezzatura da pesca. Di talché sono proprio tali ultimi prodotti contenenti plastica a mettere pesantemente a rischio gli ecosistemi marini e la biodiversità, la salute umana e quella di tutte le specie animali che si nutrono della fauna dei mari, provocando contestualmente danni economici ad attività come il turismo, la pesca e il trasporto marittimo.

A tale quadro va poi ad aggiungersi un ulteriore pericolo, quello della frammentazione dei rifiuti di plastica. Quest'ultimo fenomeno genera, infatti, la c.d. microplastica, frammenti di dimensioni inferiori a $5 \mathrm{~mm}$ che si accumulano in mare e che, proprio a causa delle minuscole dimensioni, vengono ingeriti dalla fauna marina, penetrando nella catena alimentare e diffondendosi pressoché ovunque - basti pensare che se ne è rilevata la presenza non solo in alimenti come il sale, ma persino nell'aria e nell'acqua potabile - impattando negativamente sull'ambiente e sulla salute collettiva.

Di fronte a tale problema globale - testimoniato tristemente dalle numerose immagini che circolano nella rete e che raffigurano giovani ricciole incastrate in anelli di plastica, tartarughe marine che soffocano in sacchetti di plastica confusi per meduse, ancora, cetacei rinvenuti morti sulle spiagge per l'enorme quantità di microplastica ingerita - è stata avvertita l'esigenza, a livello sovranazionale, di porre obiettivi e intraprendere misure concrete proprio al fine di ridurre l'impatto ambientale di tale diffuso materiale. Si analizzeranno tali obiettivi e misure nel paragrafo seguente.

\section{Il diritto di fronte all'emergenza: dai primi atti programmatici alla strategia europea per la plastica, fino alla nuova direttiva UE/2019/904}

Di fronte all'emergenza mondiale testé descritta - limitando lo sguardo agli interventi eurounitari ${ }^{6}$ - è intervenuta l'Unione europea, dapprima adottando atti

come illustra ancora tale comunicazione, il riciclaggio della plastica avrebbe numerosi vantaggi. Il più importante: può ridurre la dipendenza dall'estrazione di combustibili fossili (necessari per la produzione di plastica) e contenere le emissioni di $\mathrm{CO}_{2}$, consentendo di conseguire un alto risparmio energetico annuale (pari a 3,5 miliardi di barili di petrolio).

${ }^{6}$ Nonostante il presente studio si soffermi sulle misure intraprese a livello euro-unitario, è possibile analizzare tale problema anche dalla prospettiva internazionalistica. Per un approfon- 
programmatici, più recentemente con l'emanazione di atti vincolanti, come la recentissima direttiva UE/2019/904, del Parlamento europeo e del Consiglio, del 5 giugno 2019, sulla riduzione dell'incidenza di determinati prodotti di plastica sull'ambiente (atto pubblicato nella GUUE il 2 giugno 2019 e in vigore dal 2 luglio 2019).

Ripercorriamo sinteticamente le più recenti ed incisive misure intraprese a livello euro-unitario volte a ridurre l'impatto ambientale della plastica.

Sul piano prettamente programmatico, in data 2 dicembre 2015, la Commissione europea ha rivolto una comunicazione alle altre istituzioni dell'Unione europea dal titolo 'L'anello mancante - Piano d'azione dell'Unione europea per l'economia circolare", mettendo in luce la necessità di passare da un'economia lineare ad una circolare, aumentando il riciclaggio della plastica (stimato ad una soglia inferiore al $25 \%$ rispetto alla plastica prodotta in tutta Europa) e diminuendo la sua dispersione nell'ambiente, compreso quello marino ${ }^{7}$. E sulla stessa linea si pone anche la comunicazione adottata dalla Commissione in data 24 ottobre $2017^{8}$.

All'interno del Piano d'azione per l'economia circolare del 2015, poi, l'Unione europea si è altresì impegnata ad elaborare "una strategia per affrontare le sfide poste dalle materie plastiche in tutte le fasi della catena del valore e tenere conto del loro intero ciclo di vita"; strategia, questa, elaborata in data 16 gennaio 2018, quando la Commissione ha rivolto alle altre istituzioni dell'Unione europea

dimento sul quadro giuridico internazionale, cfr. in dottrina, I. Tani, Oceani di plastica: il quadro giuridico internazionale, "Rivista giuridica dell'ambiente" 2018, n. 3, pp. 613-659.

${ }^{7}$ Cfr. COM (2015) 614 final, punto 5.1, ove si afferma che "se si vuole garantire il passaggio a un'economia circolare è necessario aumentare il riciclaggio della plastica. L'uso di questo materiale nell'Unione è cresciuto in maniera costante, ma ne viene riciclato meno del $25 \%$ e circa il 50\% è collocato in discarica. Grandi quantità di rifiuti di plastica finiscono anche negli oceani, ragion per cui tra gli obiettivi di sviluppo sostenibile per il 2030 vi è quello di prevenire e ridurre in maniera significativa l'inquinamento marino di tutti i tipi, compresi i rifiuti marini. Per far sì che le materie plastiche riciclabili che attualmente finiscono nelle discariche e negli inceneritori siano invece riciclate è essenziale perfezionare i sistemi di raccolta differenziata e i regimi di certificazione delle imprese dedite alla raccolta e alla cernita". Sull'economia circolare in generale, in dottrina, cfr. fra i tanti, F. De Leonardis, Economia circolare: saggio sui suoi tre diversi aspetti giuridici. Verso uno Stato circolare?, "Diritto amministrativo" 2017, n. 1, pp. 163 ss.

${ }^{8}$ Cfr. "Programma di lavoro della Commissione per il 2018 - Un programma per un'Unione più unita, più forte e più democratica", COM (2018) 650 final. In tale comunicazione, la Commissione esprime tale volontà con le seguenti parole: "ci batteremo per sostenere l'innovazione, i posti di lavoro e la crescita attraverso la nostra strategia sull'economia circolare che può apportare immensi benefici alla nostra economia, alla nostra competitività e al nostro ambiente. Proporremo un numero limitato di proposte volte a rafforzare i nostri interventi in questo settore. Verteranno soprattutto sul modo di produrre e utilizzare le materie plastiche, nell'ottica di ottenere che tutti gli imballaggi in plastica siano riciclabili entro il 2030". 
un'altra comunicazione avente come titolo ed oggetto la "Strategia europea per la plastica nell'economia circolare"9.

Tale atto programmatico riveste assoluta centralità, essendo interamente dedicato all'utilizzo circolare di tale materiale.

Diversi sono infatti gli obiettivi posti da tale atto, fra cui: arginare la quantità di rifiuti di plastica e la loro dispersione nell'ambiente; migliorare la raccolta differenziata; incentivare la produzione della plastica con proprietà biodegradabili, all'insegna della sostenibilità; aumentare il riciclaggio della plastica (stabilendo, in particolare, che, entro il 2030, debba essere riciclata oltre la metà dei rifiuti di plastica generati in Europa); stimolare la domanda di plastica riciclata (ponendo come obiettivo la quadruplicazione della domanda di plastica riciclata in Europa, sì da garantire la stabilità del flusso di entrate per il settore del riciclaggio); orientare l'innovazione e incentivare gli investimenti verso modelli circolari (con conseguente creazione di circa 200 mila posti di lavoro in tutta Europa per attività legate al suo riciclaggio o produzione sostenibile); informare i consumatori sui benefici di tale politica e sul loro ruolo in fase di attuazione; diminuzione della dipendenza dell'Europa dalle importazioni di combustibili fossili e riduzione delle emissioni di $\mathrm{CO}_{2}$.

Recentemente, come già indicato, è stata adottata anche la direttiva UE/2019/904, atto vincolante che gli Stati membri sono tenuti a recepire entro il 3 luglio $2021^{10}$, che detta una serie di prescrizioni volte a prevenire e ridurre l'incidenza di determinati prodotti di plastica sull'ambiente acquatico e sulla salute, promuovendo la transizione verso un'economia circolare e l'adozione di modelli imprenditoriali innovativi e sostenibili ${ }^{11}$.

Tale direttiva prende in considerazione, in particolare: i prodotti di plastica monouso, quelli cioè non concepiti per essere riutilizzati o riciclati, e gli attrezzi da pesca, usati per catturare o allevare risorse biologiche marine ${ }^{12}$.

${ }^{9}$ Cfr. COM (2018) 28 final.

${ }^{10}$ Quanto ai tempi di recepimento della direttiva, è previsto che gli Stati provvedano alla sua trasposizione entro il 3 luglio 2021, salvo per quanto concerne le disposizioni relative a: il divieto di immissione sul mercato, ove il recepimento decorre dal 3 luglio 2021; i requisiti di progettazione e di etichettatura, ove il recepimento, rispettivamente, decorre dal 3 luglio 2024 e dal 3 luglio 2021; gli obblighi aggiuntivi in materia di responsabilità estesa ai produttori, in relazione alla quale il recepimento, a seconda del prodotto, deve avvenire tra il 5 gennaio 2023 e il 31 dicembre 2024.

${ }^{11}$ Cfr. art. 1, direttiva UE/2019/904.

${ }^{12}$ Cfr. art. 2, direttiva UE/2019/904. Cfr. altresì, l'art. 3 della direttiva, relativo alle definizioni. Del resto, come afferma il $7^{\circ}$ considerando della direttiva, "per concentrare gli sforzi là dove è più necessario, la presente direttiva dovrebbe considerare solo quei prodotti di plastica monouso più frequentemente rinvenuti sulle spiagge dell'Unione, come anche gli attrezzi da pesca contenenti plastica [...]. Si stima che i prodotti di plastica monouso cui si riferiscono le misure della presente direttiva rappresentino circa l' $86 \%$ dei prodotti di plastica monouso rinvenuti sulle spiagge dell'Unione". 
Diversamente, essa non ha ad oggetto le microplastiche, che, come già osservato, incidono però pesantemente sull' ecosistema marino e la salute in generale: in relazione ad essa, infatti, la direttiva si limita ad affermare solo l'opportunità che l'Unione europea incoraggi tutti i produttori a limitare rigorosamente le microplastiche nelle loro formulazioni ${ }^{13}$.

Fra le prescrizioni più importanti, la direttiva prevede l'impegno degli Stati membri di adottare le misure necessarie per conseguire, dal 2022 ed entro il 2026, una riduzione ambiziosa e duratura del consumo di alcuni prodotti di plastica monouso, come le tazze per bevande e i contenitori per alimenti destinati al consumo immediato, direttamente dal recipiente e non richiedenti ulteriore cottura o riscaldamento ${ }^{14}$. Si pensi, ad esempio, ai contenitori per fast food, scatole per pasti, per panini, per involtini, per insalate, o, ancora, contenitori per alimenti che non richiedono ulteriore preparazione, quali frutta, verdura o dolci.

Oltre ai prodotti per i quali è prevista la riduzione di consumo, la direttiva impone agli Stati membri il divieto di immissione sul mercato di ulteriori prodotti di plastica, come i bastoncini cotonati (eccetto quelli utilizzati a fini medici), le posate, i piatti, le cannucce, gli agitatori per bevande e le aste da attaccare a sostegno dei palloncini ${ }^{15}$. Detta misura, più drastica, si giustifica in base all'esigenza di imporre il ricorso ad alternative ecocompatibili e facilmente disponibili, sì da incentivare modelli imprenditoriali più sostenibili ${ }^{16}$.

${ }^{13}$ Cfr. $8^{\circ}$ considerando, direttiva UE/2019/904.

${ }^{14}$ Per i prodotti per i quali è prevista la riduzione di consumo, si veda All. A alla direttiva.

Come afferma la direttiva nella parte motivazionale, gli Stati membri dovrebbero stabilire ambiziosi obiettivi nazionali di riduzione del consumo, pur senza compromettere l'igiene alimentare, la sicurezza alimentare, le buone prassi igieniche, le buone prassi di fabbricazione, l'informazione dei consumatori o gli obblighi di tracciabilità. Cfr. 14 considerando, direttiva UE/2019/904.

In relazione all'iter procedurale, come afferma la direttiva, gli Stati sono tenuti a notificare alla Commissione, entro il 21 luglio 2021, l'elenco delle misure adottate al fine di ridurre il consumo di tali prodotti e incentivare la loro sostituzione con alternative riutilizzabili non contenenti plastica.

Dette misure, da aggiornare nel tempo, devono essere proporzionali e non discriminatorie. Quanto al loro contenuto, come suggerisce la direttiva, gli Stati dovrebbero vietare agli operatori economici di fornire, gratuitamente, al consumatore finale, presso i punti di vendita, i prodotti di plastica monouso, incentivando l'utilizzo di alternative riutilizzabili più economiche. Potendo gli Stati, inoltre, persino restringere il mercato laddove sussista la necessità, in ragione del forte impatto ambientale, di evitare che tali prodotti vengano dispersi, così incentivando la loro sostituzione con alternative riutilizzabili non contenenti plastica.

Agli stessi Stati membri spetta, infine, anche il compito di monitorare nel tempo il consumo di tali prodotti e l'efficacia delle misure attuate, aggiornando altresì la Commissione sui progressi compiuti in vista degli obiettivi quantitativi da traguardare definiti a livello euro-unitario. In relazione a tale disciplina, cfr. art. 4, direttiva UE/2019/904.

${ }^{15}$ Cfr. art. 5, direttiva UE/2019/904. Per i prodotti per i quali è previsto il divieto di immissione sul mercato, si veda l'All. B alla direttiva.

${ }^{16}$ Cfr. $15^{\circ}$ considerando, direttiva UE/2019/904. 
La direttiva detta altresì misure di progettazione in relazione ai contenitori e alle bottiglie per bevande con tappi e coperchi di plastica (utilizzati, ad esempio, per birra, vino, acqua, bibite rinfrescanti, succhi e nettari, bevande istantanee e latte): misure, queste, che valorizzano l'esigenza di tenere conto dell'intero ciclo di vita della plastica e che sono volte a ridurre, in fase di produzione, l'impiego della plastica monouso, incentivando l'utilizzo di quella riciclata ${ }^{17}$.

La direttiva detta, ancora, obblighi di etichettatura con riguardo ad assorbenti, salviette umidificate e prodotti del tabacco, dovendo informare i consumatori, con caratteri grandi, chiaramente leggibili e indelebili - e preferibilmente sull'imballaggio del prodotto oppure direttamente sul prodotto stesso - circa la presenza di plastica, le modalità di corretta gestione del prodotto, le improprie forme di smaltimento da evitare, ancora, l'incidenza negativa che la dispersione impropria del prodotto ha sull'ambiente ${ }^{18}$.

Ancora, la direttiva prevede, in linea con il principio "chi inquina paga", che gli Stati membri introducano regimi di responsabilità estesa ai produttori in relazione, appunto, alla produzione di numerosi beni di plastica, diversi da quelli per i quali è previsto il divieto di immissione sul mercato (e cioè, di fatto, quelli per i quali non sono facilmente disponibili alternative adeguate e più sostenibili): in particolare, in relazione ai contenitori per alimenti destinati al consumo immediato, tazze per bevande, contenitori e bottiglie per bevande con tappi o coperchi in plastica, sacchetti di plastica, salviette umidificate, palloncini e prodotti del tabacco ${ }^{19}$. Detto regime di responsabilità è volto a coprire i necessari costi di gestione e di rimozione dei rifiuti, nonché i costi delle misure di sensibilizzazione per prevenire e ridurre tali rifiuti ${ }^{20}$.

${ }_{17}$ Cfr. art. 6, direttiva UE/2019/904. Come afferma il $17^{\circ}$ considerando di tale direttiva, "i tappi e coperchi di plastica dei contenitori utilizzati per bevande sono tra gli oggetti di plastica monouso più frequentemente rinvenuti sulle spiagge dell'Unione. Pertanto, i contenitori per bevande che sono prodotti di plastica monouso dovrebbero poter essere immessi sul mercato solo se soddisfano determinati requisiti di progettazione che riducono in modo significativo la dispersione nell'ambiente dei tappi e coperchi di plastica [...]. Onde garantire l'uso circolare della plastica, è necessario promuovere la diffusione dei materiali riciclati sul mercato. È opportuno pertanto introdurre requisiti che prevedano un contenuto minimo obbligatorio di plastica riciclata nelle bottiglie per bevande".

${ }^{18}$ Cfr. art. 7, direttiva UE/2019/904. Cfr. altresì, in relazione ai prodotti del tabacco, il $16^{\circ}$ considerando della direttiva, secondo cui "i filtri di prodotti del tabacco contenenti plastica sono il secondo articolo di plastica monouso più frequentemente rinvenuto sulle spiagge dell'Unione. È necessario ridurre l'enorme impatto ambientale causato dai rifiuti post-consumo dei prodotti del tabacco con filtri contenenti plastica che sono gettati direttamente nell'ambiente".

${ }_{19}$ Cfr. art. 8, direttiva UE/2019/904.

${ }^{20} \mathrm{Cfr}$. $21^{\circ}$ considerando, direttiva UE/2019/904. Detti costi - come indica la direttiva - non dovrebbero superare quelli necessari per fornire tali servizi in modo economicamente efficiente. 
Vi sono ancora - continuando nell'analisi della direttiva - disposizioni che fissano obiettivi di incremento di raccolta differenziata con riguardo alle bottiglie per bevande aventi tappi o coperchi in plastica (obiettivi che ammontano, entro il 2025, del $77 \%$, e, entro il 2029, del 90\% $)^{21}$.

Gli Stati membri devono poi assumere misure di sensibilizzazione dirette ai consumatori, incentivandoli ad adottare un comportamento responsabile e volto a ridurre la dispersione dei rifiuti derivanti dai prodotti di plastica, nonché misure volte a garantire che i consumatori di prodotti di plastica e attrezzi da pesca vengano informati relativamente alle modalità di gestione e smaltimento, all'incidenza sull'ambiente della dispersione impropria del rifiuto, nonché alla disponibilità di alternative riutilizzabili ${ }^{22}$.

Completano il quadro, infine, l'obbligo annuale informativo degli Stati membri verso la Commissione in relazione alle misure assunte e ai risultati raggiunti (essenziale nel monitoraggio dei risultati in vista degli obiettivi da traguardare $)^{23}$, nonché la previsione dell'obbligo degli Stati di stabilire sanzioni effettive, proporzionali e dissuasive per gli operatori economici che non rispettano le prescrizioni nazionali adottate nel recepimento della direttiva ${ }^{24}$.

\section{Conclusioni. Uno sguardo al futuro}

Come osservato, numerose ed eterogenee sono le soluzioni giuridiche offerte dall'Unione europea nella "lotta alla plastica".

Tutte queste, però, ruotano attorno ad un'unica direttrice: la sostenibilità, che deve governare la sua produzione, il suo consumo e il suo smaltimento.

È proprio la sostenibilità, infatti, che impone di produrre tale materiale tenendo conto del suo intero ciclo di vita, evitando il monouso e incentivando il suo riciclaggio, all'insegna della circolarità.

Ciò richiede, chiaramente, tempi. Non solo quelli giuridici, indicati nei vari atti programmatici e vincolanti dell'Unione europea, ma anche e soprattutto quelli materiali, occorrendo che aumenti la domanda di plastica riciclata e, ancor prima, che venga meno l'idea che si tratti di un mercato a bassa redditività.

${ }^{21}$ Cfr. art. 9, direttiva UE/2019/904. Cfr. anche il $27^{\circ}$ considerando della direttiva, che afferma che "le bottiglie per bevande che sono prodotti di plastica monouso sono tra i rifiuti marini più frequentemente rinvenuti sulle spiagge nell'Unione. Ciò è dovuto all'inefficacia dei sistemi di raccolta differenziata e alla scarsa partecipazione dei consumatori a tali sistemi. È necessario promuovere sistemi di raccolta differenziata più efficaci. È opportuno, pertanto, fissare un obiettivo minimo di raccolta differenziata per le bottiglie per bevande che sono prodotti di plastica monouso".

${ }^{22}$ Cfr. art. 10, direttiva UE/2019/904. Cfr. anche il $28^{\circ}$ considerando della direttiva.

${ }^{23}$ Cfr. art. 13, direttiva UE/2019/904. Cfr. anche il $30^{\circ}$ considerando della direttiva.

${ }^{24}$ Cfr. art. 14, direttiva UE/2019/904. 
Non solo. Occorre, ancora, un impegno congiunto fra settore pubblico (dalle autorità nazionali a quelle regionali, e infine a quelle locali, più vicine ai cittadini) e settore privato (dai produttori di materie plastiche ai rivenditori, ai gestori del riciclaggio, fino ai consumatori), richiedendosi altresì lo sforzo degli organismi internazionali per incentivare il cambiamento oltre i confini europe ${ }^{25}$. Unica via, quest'ultima, per affrontare un problema che ha dimensioni globali.

Solo diminuendo la quantità di rifiuti di plastica e incentivando il suo riciclaggio, infatti - gettando uno sguardo al futuro - sarà possibile ridurre il terribile impatto ambientale di tale materiale.

Infatti, da un lato, verrebbe a ridursi la dipendenza dall'estrazione e importazione di combustibili fossili, con contenimento delle emissioni di $\mathrm{CO}_{2}$, dall'altro, evitando la dispersione tout court del materiale, sarebbe possibile conservare la biodiversità e le risorse marine, salvaguardando, così facendo, anche la salute umana.

Dovendosi altresì rilevare che tale nuovo approccio, oltre a spingere verso la sostenibilità e la circolarità, significherebbe, per l'industria europea della plastica, anche competitività, innovazione e occupazione.

Solo per tale via, l'Europa potrà trasformare le sfide connesse alla produzione, al consumo e alla fine del ciclo di vita della plastica in opportunità, tanto per assicurare un ambiente più sano e pulito, quanto per condurre l'industria europea della plastica alla crescita e alla prosperità, realizzando, a livello globale, un modello virtuoso da emulare.

\title{
COMBATING PLASTIC AND MARINE ECOSYSTEMS. THE LEGAL FRAMEWORK ARISING FROM DIRECTIVE (EU) NO 2019/904
}

\author{
Summary
}

The article describes, first, the global emergency related to the production, consumption and dispersion in the environment of non-recyclable or reusable disposable plastic. Subsequently, it continues with the analysis of the objectives and measures established in the context of the European strategy for plastics and the recent Directive(EU) No 2019/904. In fact, behind the objectives and legal solutions offered by the European Union, there is the hope, shared by the author, that the challenges connected to the production, consumption and the end of the life cycle of plastic can become opportunities, and will not only guarantee everyone a healthier environment, but will also drive the European plastics industry towards innovation and growth, creating at the same workplaces and increasing the rate of employment.

${ }^{25}$ In questo senso, il $35^{\circ}$ considerando della direttiva UE/2019/904. 


\title{
LOTTA ALLA PLASTICA ED ECOSISTEMI MARINI. IL QUADRO GIURIDICO ALL'INDOMANI DELLA DIRETTIVA UE/2019/904
}

\begin{abstract}
Riassunto
Nell'attuale contesto sociale, è particolarmente avvertita, a livello mondiale, l'esigenza di contenere l'impatto ambientale relativo alla produzione, consumo e dispersione nell'ambiente della plastica monouso non riciclabile o riutilizzabile. Proprio l'emergenza globale connessa alla diffusione di tale materiale viene illustrata nella prima parte del presente studio, ove ne vengono forniti anche allarmanti numeri. Successivamente, l'articolo prosegue con l'analisi degli obiettivi e delle misure stabilite nell'ambito della strategia europea per la plastica e dalla recente direttiva UE/2019/904. Dietro gli obiettivi e le soluzioni giuridiche offerte dall'Unione europea, infatti, v'è la speranza - condivisa dall'Autore - che le sfide connesse alla produzione, al consumo e alla fine del ciclo di vita della plastica possano trasformarsi in opportunità, tanto per assicurare a tutti un ambiente più sano e pulito, quanto per condurre l'industria europea della plastica all'innovazione e alla crescita, con maggiori livelli anche occupazionali.
\end{abstract}

